\title{
Automation of NLO computations using the FKS subtraction method
}

\author{
Rikkert Frederix * \\ Institute for Theoretical Physics, Universität Zürich \\ E-mail: frederix@physik.uzh.ch
}

In this talk the FKS subtraction method for next-to-leading order (in QCD) computations is reviewed and its automation in the computer code MadFKS is presented. As a proof of concept, some results in the form of plots for distributions at next-to-leading order in $e^{+} e^{-} \rightarrow 2,3$ and 4 jets are shown by interfacing this code to BlackHat and Rocket.

RADCOR 2009 - 9th International Symposium on Radiative Corrections (Applications of Quantum Field Theory to Phenomenology),

October 25 - 302009

Ascona, Switzerland

\section{* Speaker.}

†Based on Ref. [1] in collaboration with Stefano Frixione, Fabio Maltoni and Tim Stelzer. Special thanks to Daniel Maître and Giulia Zanderighi for help with interfacing MadFKS to BlackHat and Rocket, respectively. 


\section{Introduction}

In perturbation theory, next-to-leading order (NLO) predictions consist of three distinct contributions. There is the Born contribution, which is the lowest order for the process to occur, with all final state particles resolved. The second contribution is the (ultraviolet renormalized) virtual correction that has the same external particles as the Born, but with an extra power of the coupling constant. This leads to one ${ }^{1}$ internal loop of particles with a loop momentum that has to be integrated over. And thirdly, the real emission contribution that has one extra particle in the final state, which may or may not be resolved, has to be included. We can present these three contributions to a NLO prediction for a $2 \rightarrow m$ process schematically as

$$
\sigma^{\mathrm{NLO}}=\int_{m} \mathrm{~d} \sigma^{\text {Born }}+\int_{m} \mathrm{~d} \sigma^{\text {virt. }}+\int_{m+1} \mathrm{~d} \sigma^{\text {real }},
$$

where the integrals are over the $m$-particle phase space for the Born and the virtual contributions and over the $(m+1)$-particle phase space for the real emission corrections.

In the (ultraviolet renormalized) virtual corrections the loop integral over the internal momentum is divergent. Also the phase-space integral over the (possibly) unresolved particle in the real emission is divergent. These divergences are of infrared origin and only after the virtual and the real emission contributions are added together, they cancel against each other. In almost all practical calculations for NLO QCD predictions these integrations are performed using a dimensional regularization scheme in which the dimension of these integrals is shifted away from 4 , conventionally by a factor $2 \varepsilon$. After integration the divergences will show up as explicit poles in $1 / \varepsilon$ and their cancellation can be verified explicitly.

The calculation of an integral in a non-integer number of dimensions can only be done analytically. But, experimental analyses require the possibility to make complicated phase-space cuts and therefore it is an impossible task to perform the phase-space integrals by analytic means. A Monte Carlo technique is favoured. However, this numerical approach implies that the phase-space integrals have to be performed in 4 dimensions, which leads to infrared divergences for the real emission contributions.

There are two classes of solutions to this problem. They are the (approximate) phase-space slicing or the (exact) subtraction method; it is nowadays acknowledged that the slicing method is unsuited for describing complicated final states, such as those in multi-jet production. In the subtraction method a term which has the same singularity structure as the real emission corrections is added to these contributions. This cancels the infrared divergences in the phase space integral. The subtraction terms should be simple enough such that the one-particle phase-space integral (of the unresolved particle) can be done analytically in dimensional regularization. Schematically we can write this as:

$$
\sigma^{\mathrm{NLO}}=\int_{m}\left[\mathrm{~d} \sigma^{\text {Born }}+\mathrm{d} \sigma^{\text {virt. }}+\int_{1} \mathrm{~d} \sigma^{\text {subtr. }}\right]+\int_{m+1}\left[\mathrm{~d} \sigma^{\text {real }}-\mathrm{d} \sigma^{\text {subtr. }}\right] .
$$

The remaining phase-space integrals can now be performed numerically and give finite results for infrared safe observables.

In the next section we describe the code MadFKS [1] in which the subtraction method introduced by Frixione, Kunszt and Signer (FKS) [2] has been implemented. Another widely used subtraction method, originally proposed by Catani and Seymour [3], has already been implemented by several groups [4]. In Sec. 3 we shown some results for NLO predictions for event shapes in electron-positron collisions. We finish with the conclusions.

\footnotetext{
${ }^{1}$ For processes without a tree-level Born (such as Higgs production via gluon fusion), the virtual corrections have always one loop more than the Born.
} 


\section{Automated subtraction}

Before explaining some of the features of the MadFKS code, the FKS subtraction method is shortly reviewed here in a schematic way. For more detailed explanations we refer to the papers of Refs. $[1,2]$.

\subsection{FKS subtraction}

The real emission contribution in Eq. (1.1) can be written schematically as

$$
\mathrm{d} \sigma^{\text {real }}=\left|M^{m+1}\right|^{2} \mathrm{~d} \phi_{m+1},
$$

where $\left|M^{m+1}\right|^{2}$ is the matrix element squared and $\mathrm{d} \phi_{m+1}$ the phase-space measure. The matrix element squared blows up ${ }^{2}$ like $\frac{1}{\xi_{i}} \frac{1}{1-y_{i j}}$, where $\xi_{i}$ is the energy of final state particle $i$ (in the partonic centerof-mass frame) over the total partonic energy, $\xi_{i}=2 E_{i} / \sqrt{\hat{s}}$, and $y_{i j}$ is the cosine of the angle between particle $i$ and $j, y_{i j}=\cos \theta_{i j}$. The crucial step in the FKS subtraction method is to realize that the phase-space can be partitioned into regions that have at most one collinear and/or one soft divergence. This can be achieved by multiplying Eq. (2.1) by so-called $S$-functions

$$
\mathrm{d} \sigma^{\text {real }}=\sum_{i j \text {-pairs }} S_{i j}\left|M^{m+1}\right|^{2} \mathrm{~d} \phi_{m+1},
$$

where the $S$-functions have been defined in such a way that they vanish in all singular limits not related to particle $i$ becoming soft or particles $i$ and $j$ collinear and that the sum over all pairs gives one, $\sum_{i j \text {-pairs }} S_{i j}=1$. The precise definition of the $S$-functions used is not important. The result is that each term of the sum in Eq. (2.2) is finite over all of phase space except if the energy of particle $i$ goes to zero or particles $i$ and $j$ become collinear.

Because we know exactly where the infrared singularities are for a given partition, it is now relatively straight-forward to regularize these divergences. This amounts to replacing each of the terms in the sum of Eq. (2.2) by

$$
\left(\frac{1}{\xi_{i}}\right)_{\xi_{\text {cut }}}\left(\frac{1}{1-y_{i j}}\right)_{\delta_{O}} \xi_{i}\left(1-y_{i j}\right) S_{i j}\left|M^{m+1}\right|^{2} \mathrm{~d} \phi_{m+1},
$$

where we have used generalized plus distributions defined, with a smooth test function $f(x)$, as

$$
\int d x\left(\frac{1}{x}\right)_{x_{\text {cut }}} f(x)=\int d x \frac{f(x)-f(0) \Theta\left(x_{\text {cut }}-x\right)}{x} .
$$

This leads to (maximally) three counter terms for a single $(m+1)$-particle event:

- the soft counter event: $\xi_{i}=0$;

- the collinear counter event: $y_{i j}=1$;

- and the soft-collinear counter event: $\xi_{i}=0$ and $y_{i j}=1$;

Of course, for numerical evaluation the explicit $\xi_{i}$ and $\left(1-y_{i j}\right)$ in Eq. (2.3) should be canceled analytically against the divergences in the matrix element squared. But this yields no problem, because it is appreciated that in the collinear limit the real emission matrix element squared is equal to the Born times the Altarelli-Parisi splitting functions and in the soft limit by the color-linked Borns times the eikonals. This defines the last two terms of Eq. (1.2).

\footnotetext{
${ }^{2}$ The phase space measure includes an implicit factor $\xi_{i}$. It has been assumed that this has already canceled one of the $\frac{1}{\xi_{i}}$ in the matrix element squared.
} 
In order not to change the NLO prediction, the "integrated subtraction terms" need to be added to the virtual corrections, i.e., the third term in Eq. (1.2). The integral over the unresolved particle can be performed analytically and is process independent; it results in terms proportional to the (color-linked) Borns. For the explicit formulas of these integrated subtraction terms we refer to the paper of Ref. [1].

\subsection{MadFKS}

In MadFKS the generation of all the tree-level structures to a NLO prediction has been automated within the MadGraph/MadEvent [5] framework. That is, given the $(m+1)$ process as input, a Fortran code is created to analyze the Born, the real emission and the (integrated) subtraction terms. Furthermore a parametrization for the phase space is set-up that is parallel in nature and results in an efficient phase-space integration to give the results in the form of plots for distributions.

To reduce the number of phase space partitions in Eq. (2.2) and with that the total number of subtraction terms, the symmetry of the matrix elements and the phase space is used. For example, for all final state gluons only one contribution to the partition needs to be included to correctly cover the whole phase space and subtract all their corresponding singularities. This greatly reduces the growth of the number of subtractions terms with the number of external particles. In fact, only adding new (anti-)quark flavors or the first gluon increases the number of terms.

For the phase-space integration of each of the phase-space partitions, we have adopted the "singlediagram-enhanced multi-channel" integration method used by MadGraph/MadEvent. Even though the phase-space integration in the sum is over the $(m+1)$-particle phase space, we use the Born diagrams to set-up the channels. To get the $(m+1)$-particle phase space from the Born, we generate three extra random numbers related to the $\xi_{i}, y_{i j}$ and azimuth $\phi$ of the unresolved particle and perform a boost to put all the particles back on-shell. Each of the integration channels can be run on a separate computer.

Another significant improvement is achieved by reducing the number of times the Born matrix elements are computed. The Born itself, the collinear counter term, the soft counter term, the soft-collinear counter term, the three integrated subtraction terms and the enhancement factor for the multi-channel integration are all places where the Born is needed. With our choice of the phase space parametrization and carefully caching all the helicity amplitudes the Born matrix elements need to be computed only once for each $(m+1)$-particle phase space point.

For phase space partitions that have a massive particle $j$, only the soft counter event for (massless) particle $i$ needs to be included: there is no singularity in the collinear limit for particles $i$ and $j$. Due to the fact that the form of the soft singularity is independent of the spin of the particle, the implementation of the subtraction method works without modification also for all the New Physics models in MadGraph/MadEvent that include new massive QCD particles, like squarks in SUSY.

There are many internal non-physical parameters that can be "tuned" if need be: the $\xi_{\text {cut }}$ and $\delta_{O}$ that restrict the region of subtraction and the explicit form of the $S$-functions. We have checked that the final results computed by MadFKS do not depend on these internal parameters. Also the integration uncertainty does not dependent on the values of these parameters within reasonable ranges. We therefore conclude that the implementation is correct and the integration over the phase space wellunderstood.

Contrary to the Born, the real emission and the subtraction terms that are automated in MadFKS, the fully differential (finite parts of the) virtual corrections have to be provided by the user. MadFKS follows the proposal for the Binoth Les Houches Accord for the interface between Monte Carlo and one-loop programs [6]. Therefore, if the user provides a code in the form of a library with a subroutine 

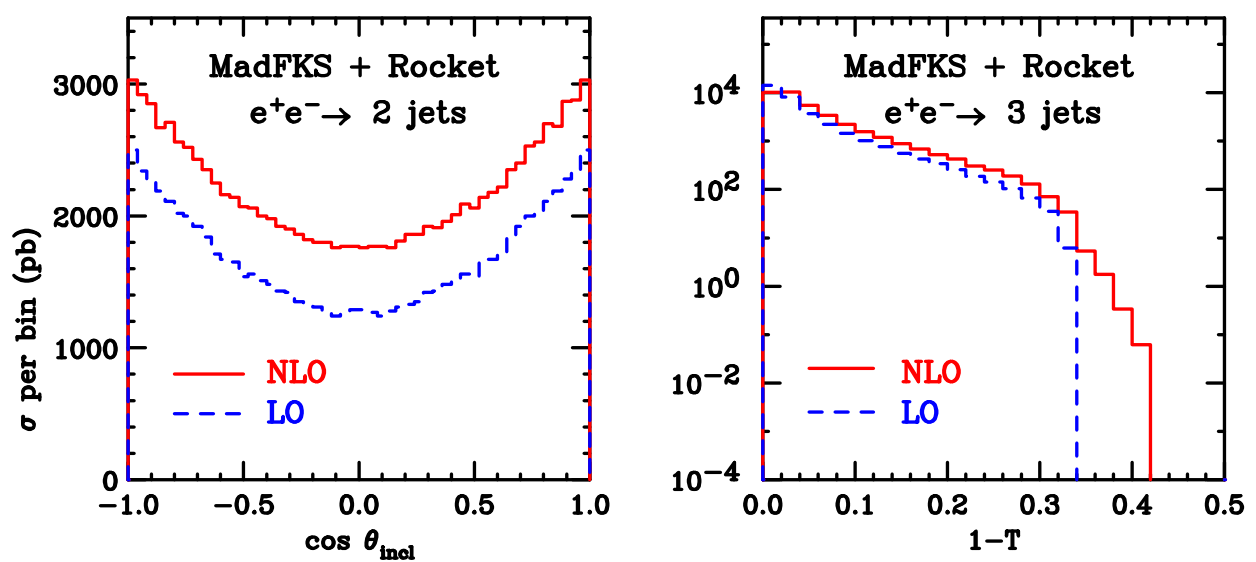

Figure 1: Inclusive $\cos \theta$ for 2 jet production and Thrust distribution for 3 jet production at LO (blue dashed) and NLO (red solid) using MadFKS and Rocket.

that returns the size of the virtual corrections for a given phase-space point as prescribed by the Binoth LHA proposal, it can easily be linked to MadFKS. For the moment, this interface has been tested with the BlackHat [7] and Rocket [8] one-loop programs.

So far, in MadFKS only the soft and final-state collinear subtraction terms have been implemented. Although the formulas for the remaining (initial state collinear) subtraction terms have all been worked out and implemented, this part of the code is still in testing phase and not yet ready to produce physics results.

\section{Selected results}

As a proof of concept we show here some selected results for NLO corrections to electron-positron collisions to 2,3 and 4 jets at $\sqrt{\hat{s}}=M_{Z}$ and with the renormalization scale also equal to the $Z$ boson mass.

In Fig. 1 results for Rocket linked to MadFKS are plotted. On the left hand side are the LO and NLO predictions shown for the inclusive $\cos \theta$ distribution in 2 jets production. This distribution is defined as the cosine of the angle between the incoming electron direction and all of the final state jets, defined according to the Durham jet algorithm and using the E-scheme to recombine the momenta, i.e., we add the particles four-momenta. On the right hand side are the fixed LO and NLO predictions shown of (one minus) the thrust distributions, which starts from Born-level 3 parton events and is therefore shown for 3-jet events.

In Fig. 2 we show two distributions calculated by linking BlackHat code to the MadFKS MC program. In the plot on the left hand side, the $C$ parameter is shown in $e^{+} e^{-} \rightarrow 3$ jets at LO and NLO, and in the plot of the right hand side the $D$ parameter in $e^{+} e^{-} \rightarrow 4$ jets.

\section{Conclusions}

For any NLO computation in QCD, both in the SM as well as BSM, MadFKS takes care of generating the Born, Real emission, (integrated) subtraction terms, phase-space integration and the overall management of symmetry factors, subprocesses combination, etc. The only piece that the user should provide is the (fully-differential) finite part of the one-loop contributions. So far, there are tested and 

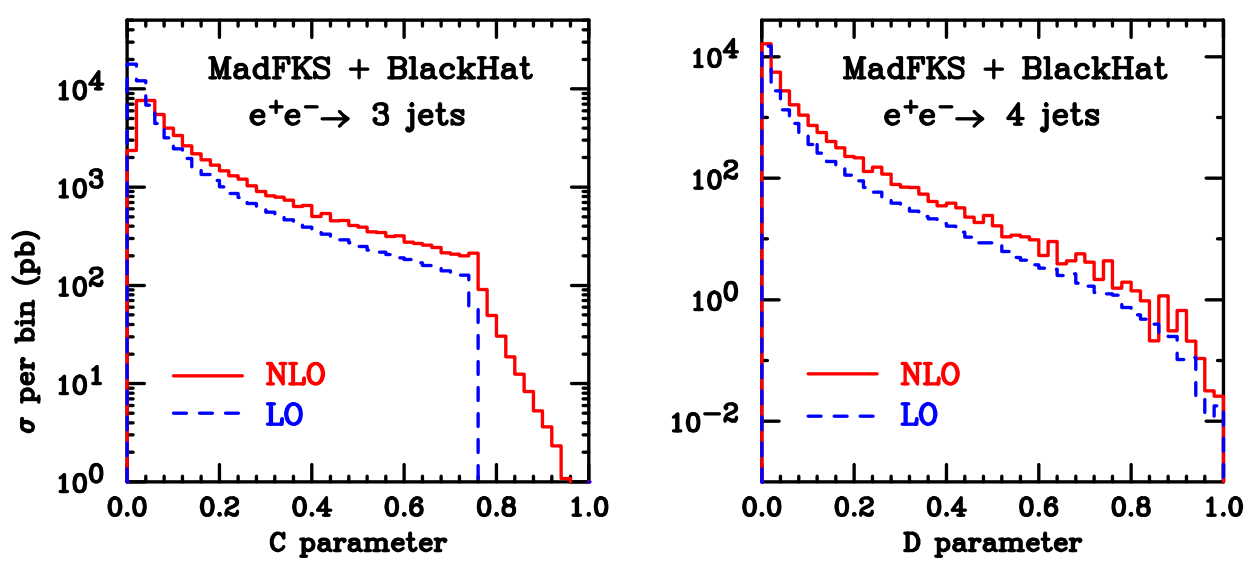

Figure 2: $C$ parameter for 3 jet production and $D$ parameter for 4 jet production at LO (blue dashed) and NLO (red solid) using MadFKS and BlackHat.

working interfaces to the BlackHat and Rocket codes following the proposal for the Binoth Les Houches Accord. As a proof of concept, we have shown results for NLO predictions for $e^{+} e^{-} \rightarrow 2,3$ and 4 jets in the form of distributions for some event shapes.

\section{References}

[1] R. Frederix, S. Frixione, F. Maltoni, and T. Stelzer, JHEP 10 (2009) 003.

[2] S. Frixione, Z. Kunszt, and A. Signer, Nucl. Phys. B467 (1996) 399-442; S. Frixione, Nucl. Phys. B507 (1997) 295-314.

[3] S. Catani and M. H. Seymour, Nucl. Phys. B485 (1997) 291-419; S. Catani et. al., Nucl. Phys. B627 (2002) 189-265;

[4] T. Gleisberg and F. Krauss, Eur. Phys. J. C53 (2008) 501-523; M. H. Seymour and C. Tevlin, arXiv:0803.2231 [hep-ph]; K. Hasegawa, S. Moch, and P. Uwer, 0911.4371 ; R. Frederix, T. Gehrmann, and N. Greiner, JHEP 09 (2008) 122; M. Czakon, C. G. Papadopoulos, and M. Worek, JHEP 08 (2009) 085.

[5] T. Stelzer and W. F. Long, Comput. Phys. Commun. 81 (1994) 357-371; F. Maltoni and T. Stelzer, JHEP 02 (2003) 027; J. Alwall et. al., JHEP 09 (2007) 028.

[6] T. Binoth et al., arXiv:1001.1307 [hep-ph] .

[7] C. F. Berger et. al., Nucl. Phys. Proc. Suppl. 160 (2006) 261-270; C. F. Berger et. al., Phys. Rev. D78 (2008) 036003; C. F. Berger et. al., Nucl. Phys. Proc. Suppl. 183 (2008) 313-319.

[8] K. Melnikov and M. Schulze, JHEP 08 (2009) 049; R. Keith Ellis, K. Melnikov, and G. Zanderighi, Phys. Rev. D80 (2009) 094002; K. Melnikov and G. Zanderighi, arXiv:0910.3671 [hep-ph] . 